\title{
Pseudotumor Treated with Two-Stage Revision Due to Aggressive Osteolysis and Soft-Tissue Mass Following Metal on Metal Total Hip Arthroplasty
}

\begin{abstract}
Keywords: Pseudotumor; Total hip arthroplasty (THA); Metal on metal (MOM); Revision surgery; Mega prosthesis; custom implants
\end{abstract}

\begin{abstract}
Introduction: This is a unique case of the largest reported pseudotumor in literature, treated with a custom acetabular component in conjunction with a total femur replacement.

Case: Patient is a 71-year-old male, with a large pseudotumor secondary to Metal on Metal total hip arthroplasty, who had chronic pain for 8 years prior to presentation. Imaging revealed extensive soft tissue mass and osteolysis. Patient underwent two-stage surgery, with 1st stage involving surgical debridement of pseudotumor and fixation of custom triflange acetabular component, 2nd stage involved a total femur replacement.
\end{abstract}

Conclusion: This case demonstrates the evaluation and management of a large pseudotumor. A two-stage revision in complex pseudotumor cases, with complete revision of the implants and excision of the pseudotumor, to achieve clinical improvement and decrease complications is a viable option.

\section{Introduction}

There are multiple bearing surfaces available for Total Hip Arthroplasty (THA), with Metal On Metal (MoM) implants being a common choice [1]. MoM implants were developed for better volumetric wear propertiesand improved stability [2]. However, MoM implants have high rates of metallosis and pseudotumor formation, $1-39 \%$ of cases [1,2].

Pseudotumors are defined as non-neoplastic, non-infectious cystic or solid mass forming lesions1. In many cases they develop due to biological reactions similar to hypersensitivity type 4 reactions to metal ions, released from abrasive wear of the prosthesis, leading to osteolysis and soft-tissue damage [3]. Clinical presentation may vary from absence of symptoms to pain associated with rashes, instability, nerve palsy and prosthetic loosening with osteolysis [4].

\section{Journal of Orthopedics \& Rheumatology}

\author{
Yakkanti RR*, Nguyen D and Pretell-Mazzini J \\ Department of Orthopaedic Surgery, University of Miami, USA
}

*Address for Correspondence

Yakkanti RR, Department of Orthopaedic Surgery, University of Miami, Miller School of Medicine, 1611 NW 12th Ave \#303, Miami, FL 33136, USA, Tel: 502-689-3127; E-mail: ramakanth.yakkanti@jhsmiami.org

Submission: 11 September 2020

Accepted: 02 November 2020

Published: 16 November 2020

Copyright: ๑ 2020 Yakkanti RR, et al. This is an open access article distributed under the Creative Commons Attribution License, which permits unrestricted use, distribution, and reproduction in any medium, provided the original work is properly cited.

Classification systems for stratification, diagnosis and management pathways for implant failure and soft tissue complications due to pseudotumors have been described in literature; however, no current evidence-based surgical management guideline exists $[5,6]$.

We describe a patient with a failed MoMTHA due to a large fibroblastic pseudotumor causing osteolysis of the left hemipelvis and femur. To our knowledge, this is the first case report in which a custom tripflange acetabular implant in conjunction with total femur mega prosthesis was used during a two-stage revision for treatment of a pseudotumor.

The patient was informed that data concerning the case would be submitted for publication, and he consented.

\section{Case Report}

A 71-year-old male with BMI of 27.7 and history of a left MoMTHA in 1999 presented to our institution with 8 years of chronic, progressive left groinand thigh pain which was worse with weight-bearing and at night. He had complete loss of hip and knee range of motion and required a walker for ambulation. Surgical history includes left acetabular liner and femoral head exchange, without relief of symptoms, 3 years prior to presentation. Radiographs showed extensive osteolysis surrounding the acetabular implant with posterolateral subluxation of the femoral head, as well as severe osteolysis involving the femoral shaft with multiple large
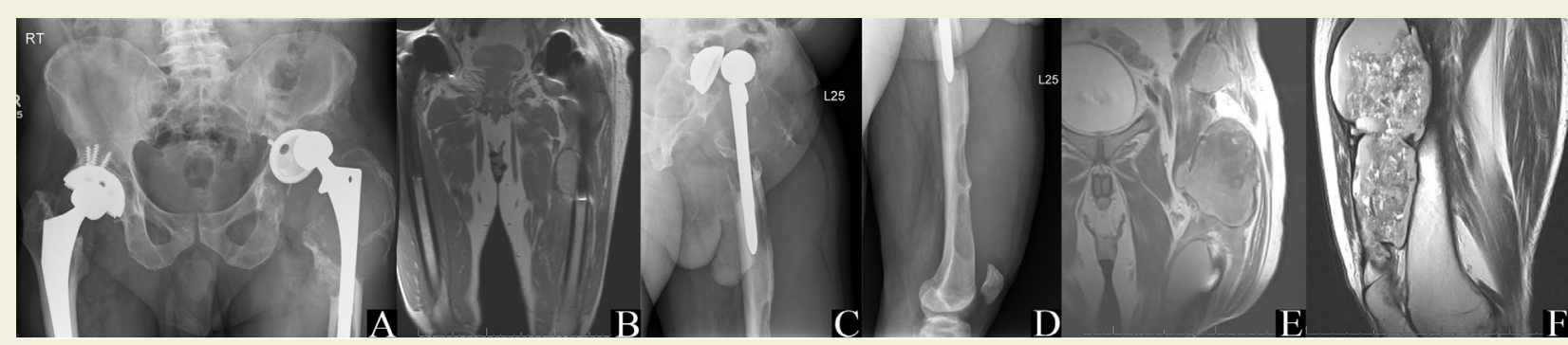

Figure 1: Pre-operative imaging. A, C, D: Plain radiographs of the pelvis and femur showing extensive osteolysis.B, E, F: MRI T2 weighted images showing the large pseudotumor and soft tissue burden surrounding the pelvis, acetabulum and femur. 
Citation: Yakkanti RR, Nguyen D, Pretell-Mazzini J. Pseudotumor Treated with Two-Stage Revision Due to Aggressive Osteolysis and Soft-Tissue Mass Following Metal on Metal Total Hip Arthroplasty. J Orthopedics Rheumatol. 2020; 7(1): 3.

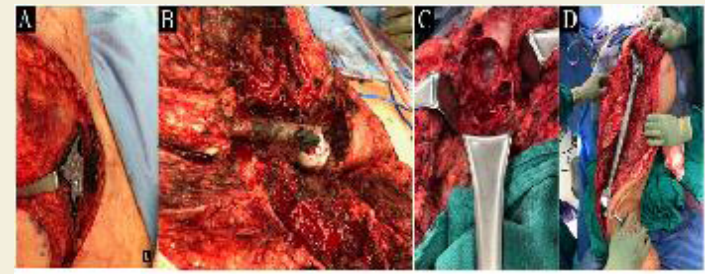

Figure 2: Intra-operative pictures. A, B: Oil like fluid which was enclosed in the pseudotumor capsule, as well as the bony osteolysis caused by the pseudotumor. C: Pelvis after final debridement and irrigation. D: Total femur constructs.

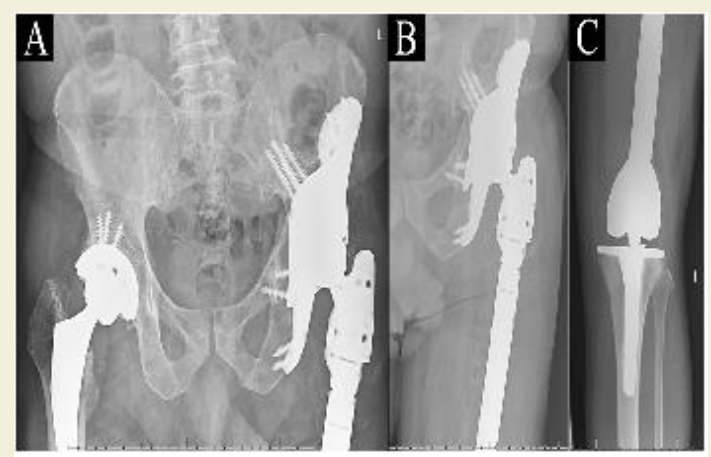

Figure 3: Post-operative imaging. A-C: Immediate post-operative plain radiographs of the implants.

lytic lesionsextending to the distal femur (Figure 1A-D). The MRI demonstrated a heterogeneous mass with layering of contents, a low signal intensity mass invasion into the ilium, large soft tissue mass on the anterior aspect of the thigh with cortical erosion and breakthrough into several points along the anterior cortex of the distal femur (Figure 1B,C,F). Laboratory results including CBC, BMP, ESR, $\mathrm{CRP}$, Serum chromium and cobalt levels were within normal limits. The osteolytic nature of the soft tissue mass prompted biopsy, which ruled out neoplastic or infectious process.

Due to the complexity of the necessary reconstruction the surgery was staged. First stage involved complete debridement of the pseudotumor surrounding the left hip, pelvis and proximal thigh using a Kocher-Langenbeck approach with proximal extension to iliac crest, and distal extension to lateral approach to femur. Dissection of the fascia lata revealed a viscous, black, oil-like fluid (Figure 2A), contained in the pseudotumor capsule. Large portions of the hip abductors and quadriceps muscles were non-viable due to metallosis. There was a "pseudo-membrane" covering the proximal thigh, with severe osteolysis of the proximal femur, acetabulum and iliac bone (Figure $2 \mathrm{~B}$ ). The pseudo-membrane was debrided, and the operative field was copiously irrigated (Figure 2C). Reconstruction of the acetabulum with a custom patient-matched triflange acetabular component was performed. (Zimmer/Biomet; Warsaw, IN.)

Three days later the second stage involving, resection of the distal two thirds of the femur due to severe bone loss, and subsequent reconstruction with a total femoral replacement(Zimmer/Biomet; Warsaw, IN) using a constrained liner in the hip and a standard rotating-hinge for the knee, was performed (Figure 2D and 3A-C).
Final pathology was compatible with a septic lymphocytic vasculitisassociated lesion (ALVAL)and inflammatory pseudotumor (Figure $4)$.

Post-operatively, the patient was allowed full weight bearing with a hip abduction brace that was discontinued at 6 weeks. At his recent follow up (14 months post-op) the patient had a Harris hip score of 82 , which is compatible with a successful outcome. There was no radiographic evidence of osteolysis or component loosening (Figure $5 \mathrm{~A}-\mathrm{C})$. Blood chromium and cobalt ions were within normal limits.

\section{Discussion}

Pseudotumors are mass forming lesions occasionally associated with MoM THA, and are described as non-infectious, non-neoplastic soft-tissue proliferations, they can display variable morphology, including solid, cystic or mixed lesions. These lesions are due to immune mediate reactions that trigger a cytokine cascade similar to type- 4 hypersensitivity reactions to metal ions more accurately described as ALVALs.2Local toxicity of the metal ions causes subsequent osteolysis and soft-tissue cell and fiber damage increasing the inflammatory stimuli.Schmalzried and Callaghan reported that these aggressive inflammatory stimuli could lead to lysis occurring at any point along the space of the hip joint [7].

In patients with pseudotumors, clinical symptoms do not necessarily correlate with the size of a pseudotumor found on MRI or ultrasound imaging [7-9]. Elevated serum cobalt might have an association with tumor size, but not necessarily to symptoms, as 57$78 \%$ of pseudotumor cases are asymptomatic $[1,2,5]$.

Pseudotumors with Cobalt (Co) and Chromium (Cr) levels of $>7$ ppbare concerning and $>20 \mu \mathrm{g} / \mathrm{L}$, with or without symptoms, receive serious consideration for revision arthroplasty $[2,3]$. Metal

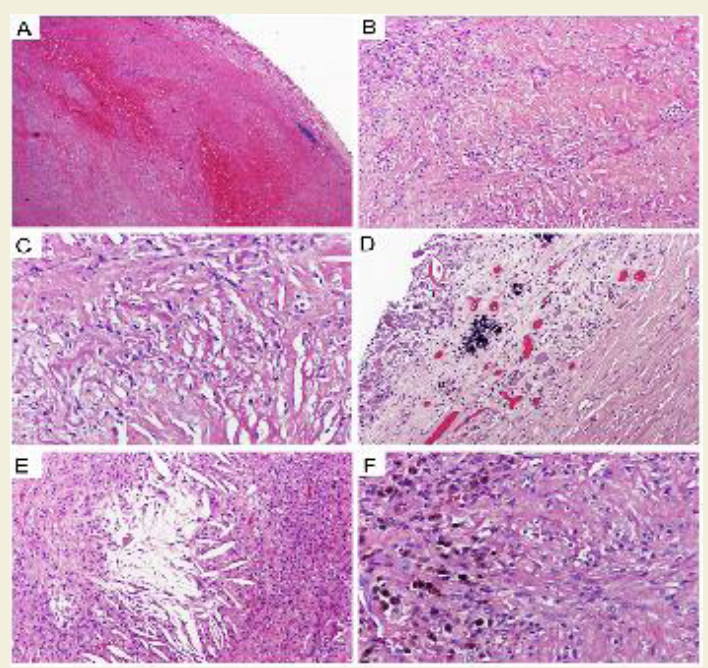

Figure 4: Pathology slides. A well circumscribed fibroblastic proliferation (A, HE 2x) with extensive collagenization ( $B$, HE 4x) admixed with lymphoplasmacytic and histiocytic inflammation (C, HE 4x) was recognized. Metal particles, cement, bone, (D, HE 10x), methacrylate fragments, and cholesterol crystals (E, HE 20x) were seen embedded. In addition, hemosiderin deposits were also present ( $F, H E$ 20x). A diagnosis of inflammatory pseudotumor and aseptic lymphocytic vasculitis-associated lesion was made. 


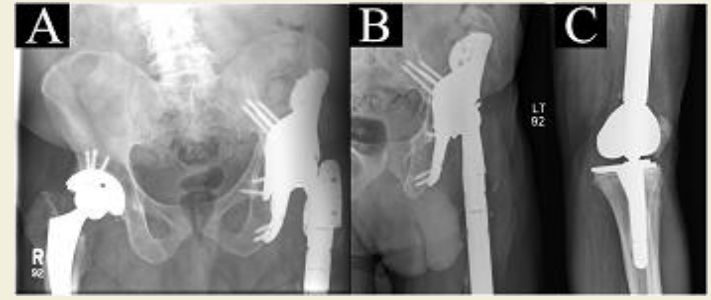

Figure 5: 14 Month follow-up imaging. A- C: plain radiographs of the implants at 14 months follow up visit.

(chromium and cobalt) ions and particles released from implants may lead to devastating cardiac and neurological effects, osteolysis, as well as soft tissue damage [10]. Risk factors for the formation of a pseudotumor are cobalt levels $>5 \mu \mathrm{g} / \mathrm{l}$, female gender, pain, and a high acetabular inclination angle $>55^{\circ}$ [8]. In our case a massive pseudotumor process with extensive involvement of bone stockin the pelvis and femur developed. This is despite normal levels of metal ionsmeasured andappropriate positioning of implants confirmed on imaging.

Treatment options include isolated revision of the femoral or acetabular component, or complete-revision surgery [10]. In many cases treatment can be performed with the same principles used in revisions for aseptic loosening. Cementless cups and femoral implants can often be used in combination with cancellous bone grafting if the bony defects are not significant. Usually the choice for revision techniques and implants is dependent on the extent of bony erosion. However, literature to guide surgical planning of pseudotumors is limited, especially with large lesions involving extensive bone and soft tissue damage. Established guidelines for non-pseudotumor revision THA do not provide adequate guidance. The treatment of this condition is associated with high reintervention rates requiring a second revision in almost $35 \%$ of the cases. Major complications occur in up to $50 \%$ of cases, with patients often experiencing the same level of pain and functional limitations as prior to the revision surgery [3].

Another important consideration is the choice of implant surfaces used in revision surgery. It is generally advisable to avoid using a metal on metal coupling when performing a revision for pseudotumor, options of ceramic on polyethylene are preferred, as they pose the lowest risk of producing metal ion debris.

This case report details a pseudotumor where the soft tissue component, and the associated osteolysis wereextensive, making this one of the largest pseudotumors reported in literature. Due to the size of the pseudotumor a unique staged surgical plan had to be implemented. Although traditionally revisions are performed in a single stage, evaluating the imaging pre-operatively gave the authors insight into the extent of osteolysis, as well as soft tissue reaction which caused a large pseudotumor to form. There was concern preoperatively that the amount of dissection required to thoroughly debride the pseudotumor would be extensive. Especially at the distal aspect of the thigh where the capsule was abutting the neurovascular bundle.

Staging was thought to decrease surgical complications, mainly infection due to reduction of surgical time in one anesthesia episode
$[4,10]$. Custom implants were indicated in order to address the irregular and extensive areas of bone loss. Due to the damaged abductor muscles, a constrained liner was used to decrease the risk of dislocation. Aggressive release of the fibrosis at the knee joint was also performed at the time of the standard total femur replacement procedure, providing improved knee range of motion. The utilization of both a total femur replacement as well as a custom triflange acetabular implant was unique in this setting and required extensive planning and coordination of care. Due to the potentially extensive nature of the dissection for both the acetabular component fixation as well as the total femur replacement and the inherent difficulty in performing these procedures in deficient bone, a more conservative approach with staging the procedure in order to reduce potential complications was thought to be appropriate.

With this case report, the authors emphasize the importance of approaching an extensive pseudotumor with careful planning and attention to detail. Complete characterization of the lesion preoperatively is especially important in order to guide appropriate surgical approach, and implant choices, including custom implants when necessary.

Case reports such as these are vital in providing practicing surgeons and researchers with an important fund of knowledge in unique cases, where large volume research studies are difficult to perform. Case series and case reports make up the majority of the available literature in regard to revision surgery secondary to pseudotumor in total hip arthroplasty, specifically in cases involving extensive bone loss and large volume formation of pseudotumor. As there are no well documented guidelines to treatment in these scenarios, this case report and others can serve as an example for a successful treatment option.

\section{Conclusion}

We recommend a two-stage revision in large complex pseudotumor cases with complete revision of the implants as well as excision of the pseudotumor to achieve clinical improvement and decrease complications. To our knowledge, this is the first case report in which a custom triphalangeal acetabular implant in conjunction with a total femur reconstruction was done with a two-stage revision for treatment of a pseudotumor due to MoM THA.

\section{References}

1. Davis DL, Morrison JJ (2016) Hip Arthroplasty Pseudotumors: Pathogenesis, Imaging, and Clinical Decision Making. J Clin Imaging Sci 6: 17.

2. Sutphen SA, MacLaughlin LH, Madsen AA, Russell JH, McShane MA (2016) Prevalence of pseudotumor in patients after metal-on-metal hip arthroplasty evaluated with metal ion analysis and MARS-MRI. J Arthroplasty 31: 260263.

3. Santavirta S, Konttinen YT, Bergroth V, Eskola A, Tallroth K, et al. (1990) Aggressive granulomatous lesions associated with hip arthroplasty. Immunopathological studies. J Bone Joint Surg Am 72: 252-258.

4. Cottino U, Dettoni F, Risitano S, Marmotti A, Rossi R (2017) Two- Total stage treatment of a large pelvic cystic pseudotumor in a Metal-on-Metal Hip arthroplasty. Joints 5: 121-124.

5. Bolognesi MP, Ledford CK (2015) Metal-on-Metal total Hip arthroplasty: patient evaluation and treatment. J Am Acad Orthop Surg 23: 724-731.

6. Schmalzried TP, Callaghan JJ (1999) Wear in total hip and knee replacements. J Bone Joint Surg Am 81: 115-136. 
Citation: Yakkanti RR, Nguyen D, Pretell-Mazzini J. Pseudotumor Treated with Two-Stage Revision Due to Aggressive Osteolysis and Soft-Tissue Mass Following Metal on Metal Total Hip Arthroplasty. J Orthopedics Rheumatol. 2020; 7(1): 3

7. Hayter CL, Potter HG, Su EP (2011) Imaging of metal-on-metal hip resurfacing. Orthop Clin North Am 42: 195-205, viii.

8. Muraoka K, Naito M, Nakamura Y, Hagio T, Takano K (2015) Usefulness of ultrasonography for detection of pseudotumors after metal-on-metal total hip arthroplasty. J Arthroplasty 30: 879-884.
9. Frisch NB, Wessell NM, Taliaferro K, Van Holsbeeck M, Silverton CD (2017) Ultrasound findings in asymptomatic patients with modular metal on metal total hip arthroplasty. Skeletal Radiol 46: 641-649.

10. Wang J, Wang D, Du J, Lin Z (2017) A new strategy to reconstruct type III acetabular bone defect associated with inflammatory pseudotumor: combined medial and lateral acetabular bone grafting: A case report. Medicine (Baltimore) 96: e8777. 